\title{
STAT3 acetylation-induced promoter methylation is associated with downregulation of the ARHI tumor-suppressor gene in ovarian cancer
}

\author{
JIE LI $^{1^{*}}$, GENG CUI $^{2 *}$, LU SUN $^{3 *}$, SHU-JUAN WANG ${ }^{1}$, YA-LI LI ${ }^{1}$, YUAN-GUANG MENG ${ }^{1}$, \\ ZHENG GUAN ${ }^{1}$, WEN-SHENG FAN ${ }^{1}$, LI-AN LI ${ }^{1}$, YI-ZHUO YANG ${ }^{1}$, YAN-QIN YOU ${ }^{1}$, \\ XIAO-YU FU ${ }^{1}$, ZHI-FENG YAN $^{1}$ and $\mathrm{KE} \mathrm{HUANG}^{1}$ \\ Departments of ${ }^{1}$ Gynecology and Obstetrics, ${ }^{2}$ Orthopedics, and ${ }^{3}$ Pathology, \\ General Hospital of PLA, Beijing 100853, P.R. China
}

Received February 1, 2013; Accepted April 2, 2013

DOI: $10.3892 /$ or.2013.2414

\begin{abstract}
ARHI is a Ras-related imprinted tumor-suppressor gene that inhibits cancer cell growth and motility. ARHI is downregulated in the majority of ovarian cancer cells, and promoter methylation is considered to be associated with its loss of expression. However, the underlying mechanisms are not well understood. Thus, the present study aimed to investigate the specific functions of ARHI and its methylation in ovarian cancer cell proliferation. Furthermore, we examined the possible role of acetylated STAT3 in modulating the expression of ARHI and its methylation. In accordance with the majority of previous studies, reduced ARHI expression was found in epithelial ovarian cancer tissues and cancer cell lines as indicated by immunohistochemistry and RT-PCR. In addition, $\mathrm{CpG}$ islands I and II within ARHI promoter regions were partially methylated or hypermethylated in cancer cell lines (SKOV-3 and HO-8910) as analyzed by pyrosequencing assays, resulting in enhanced proliferation of the cancer cells. This proliferation was reversed by the administration of 5-aza-2'-deoxycytidine. Subsequently, we demonstrated that STAT3 acetylation was increased in HO-8910 cells, and the methylation status of $\mathrm{CpG}$ I was altered in response to the acetylation of STAT3 using western blotting. Finally, chromatin immunoprecipitation (ChIP) and IP analysis indicated that acetylated STAT3 bound to the ARHI promoter and recruited DNA methyltransferase 1 for genetic modification. In conclusion, acetylated STAT3-induced promoter gene
\end{abstract}

Correspondence to: Dr Ya-Li Li or Dr Yuan-Guang Meng, Department of Gynecology and Obstetrics, General Hospital of PLA, Beijing 100853, P.R. China

E-mail: yalilibeijing@163.com

E-mail: yuanguang_meng@163.com

${ }^{*}$ Contributed equally

Key words: ARHI, promoter methylation, STAT3, acetylation, DNMT1 methylation accounts for the loss of ARHI expression and cancer cell proliferation.

\section{Introduction}

ARHI, first described in 1999 by Yu et al using differential display PCR (1), is a maternally imprinted tumor-suppressor gene located on chromosome $1 \mathrm{p} 31$ that encodes a $26-\mathrm{kDa}$ small GTP-binding protein sharing 54-62\% amino acid homology with Ras and Rap (2,3). Rather than acting as a proto-oncogene similar to ras and rap, ARHI is responsible for inducing apoptosis and inhibiting cancer cell growth and motility $(4,5)$. It has been reported that ARHI is expressed consistently in primary ovarian and breast epithelial cells but is dramatically downregulated in the majority of ovarian, breast, and even pancreatic and hepatocellular cancer cells (6). Subsequently, loss of heterozygosity (LOH) of ARHI was found in $\sim 40 \%$ of ovarian cancer cells. Multiple factors appear to contribute to the abnormal gene conversion (2). Although the definite molecular pathogenesis of $\mathrm{LOH}$ has not been elucidated, oxidative stress is suggested to be associated with the LOH-mediated carcinogenesis of ovarian cancer (7). On the other hand, promoter methylation-induced ARHI silencing is also critical to downregulated expression of ARHI.

Three potential CpG islands in the ARHI gene of 300 bp each have been identified by genomic structure analysis. Two of them (CpG islands I and II) are located within the promoter and adjacent exon 1 of the ARHI gene, while $\mathrm{CpG}$ island III is located in the protein-encoding region of exon 2 (8). Accumulating evidence indicates that the promoter methylation status is closely related to reduced ARHI expression and malignant proliferation of ovarian cancer. Feng et al (9) reported that ARHI CpG islands I and II are hypermethylated in 31 and $12 \%$ of ovarian cancers, respectively. However, how ARHI is methylated and regulated requires further investigation.

STAT3, signal transducer and activator of transcription 3, a well-known transcription activator for many genes, has been reported to inhibit gene expression. The importance of phosphorylation in regulating STAT3 functions has been the 
subject of intense scrutiny; at the same time, acetylation of STAT3 in mediating cancer progression is gradually surfacing and has attracted increased attention (10-12). STAT3 has been shown to increase methylation of $\mathrm{CpG}$ islands in certain tumorsuppressor genes through regulation of the expression and interaction with DNA methyltransferase 1 (DNMT1), which plays key roles in maintenance of the methylation status as well as inhibition of tumor-suppressor genes through aberrant CpG island methylation. Thus, dysregulation of STAT3 could relate, in part, to loss of function of critical tumor-suppressor genes, which may exaggerate the malignant proliferation and invasiveness of ovarian cancer cells.

To date, ovarian cancer still ranks third in incidence and first in mortality among all gynecological malignancies worldwide, of which epithelial ovarian cancer (EOC), including endometrioid adenocarcinoma (EAC), clear cell carcinoma (CCC) and other types, is the leading cause of cancer-related death in women, with a 5-year survival rate less than $20 \%(13,14)$. Therefore, understanding the underlying mechanisms of cellular carcinogenesis and apoptosis regulation are of paramount importance. Our study was carried out to investigate the specific functions of ARHI and its methylation in ovarian cancer cell proliferation. Furthermore, we examined the possible role of acetylated STAT3 in modulating the expression of ARHI and its methylation, which may be an important therapeutic strategy for treating ovarian cancer.

\section{Materials and methods}

Cell lines, tissue samples and regents. The human ovarian cancer cell lines (SKOV3 and HO-8910) were obtained from the American Type Cell Culture Collection (ATCC, Manassas, VA, USA), and early passages of normal human ovarian epithelial cell lines (NOE095 and HOSEpiC) were purchased from ScienCell Research Laboratories (San Diego, CA, USA) and deemed free of mycoplasma and bacterial contaminants. The SKOV3 and HO-8910 cells were maintained in RPMI1640 medium supplemented with $10 \%$ heat-inactivated FBS at $37^{\circ} \mathrm{C}$ with $5 \% \mathrm{CO}_{2}$, while the NOE095 and HOSEpiC cells were cultivated in Ovarian Epithelial Cell Medium (OEpiCM) obtained from ScienCell Research Laboratories under the same conditions. Furthermore, an HO-8910 cell line harboring the endogenous STAT3 K685R mutation was established as previously described (15) using a homologous recombinationmediated knock-in strategy.

Twenty pairs of matched ovarian cancers and normal tissues from the same patients (mean age $38 \pm 7$ years) were obtained from the Department of Gynecology and Obstetrics, at General Hospital of PLA, between January 2011 and April 2011. All tissues and brushings were fresh-frozen and stored at $-80^{\circ} \mathrm{C}$. The present study was approved and monitored by the Ethics Committee of the General Hospital of PLA.

5-Aza-2'-deoxycytidine (5-Aza), resveratrol and trichostatin were purchased from Sigma-Aldrich, and administered to the cell cultures at $0.5-1 \mu \mathrm{M}, 500 \mathrm{ng} / \mathrm{ml}$ and $10 \mu \mathrm{M}$, respectively.

Immunohistochemical staining. Paraffin-embedded normal ovarian epithelium tissues and ovarian cancer tissues were sectioned and mounted on polylysine-coated slides, dewaxed in xylene and rehydrated using a graded ethanol series, before being washed three times with phosphate-buffered saline (PBS). After initial deparaffinization, endogenous peroxidase activity was blocked using $0.3 \%$ hydrogen peroxide, and the sections were steamed in 1X Diva Decloaker (Biocare Medical, Concord, CA, USA) for $1 \mathrm{~h}$ to restore latent epitopes. The slides were then incubated with an anti-ARHI antibody (C-12) obtained from Santa Cruz Biotechnology, Inc. (Santa Cruz, CA, USA) at a final concentration of $15 \mu \mathrm{g} / \mathrm{ml}$ for $3 \mathrm{~h}$ at $37^{\circ} \mathrm{C}$. Thorough washing in $0.01 \mathrm{~mol} / \mathrm{l} \mathrm{PBS}$ was then performed. The slides were incubated for $30 \mathrm{~min}$ with each biotin-labeled secondary antibody, and then with streptavidin/peroxidase. Subsequently, after administration of diaminobenzidine substrate, the tissue sections were lightly counterstained with hematoxylin for $\sim 40 \mathrm{sec}$ and then fixed with neutral balata for observation.

Real-time RT-PCR. RT-PCR was performed to investigate the expression of ARHI. Total RNA of all normal ovarian epithelial cells (NOE095 and HOSEpiC) and cancer cells (SKOV3 and HO-8910) as well as normal and cancerous tissues were extracted and purified using an RNeasy Mini kit according to the manufacturer's instructions (Qiagen, Santa Clarita, CA, USA). After assessing RNA purity, $5 \mu \mathrm{g}$ of RNA was used as the template for PCR. ARHI primers (sense 5'-TCTGCCC GCCCTGCTTAT-3' and antisense 5'-TTGCCGTCGCCACTC TTG-3') were used with GAPDH primers (sense 5'-GCCAAA AGGGTCATCATCTC-3' and antisense 5'-GTAGAGGCAG GGATGATGTTC-3') as the internal control. Amplification cycles consisted of $94^{\circ} \mathrm{C}$ for $3 \mathrm{~min}$, then 33 cycles at $94^{\circ} \mathrm{C}$ for $1 \mathrm{~min}, 58^{\circ} \mathrm{C}$ for $1 \mathrm{~min}$, and $72^{\circ} \mathrm{C}$ for $1.5 \mathrm{~min}$, followed by $72^{\circ} \mathrm{C}$ for $15 \mathrm{~min}$. Aliquots of the PCR products were electrophoresed on $1.5 \%$ agarose gels, and PCR fragments were visualized by ethidium bromide staining.

Methylation analysis of the ARHI CpG islands. Pyro-sequencing analysis performed as previously described (16) was used to measure the ARHI promoter $\mathrm{CpG} \mathrm{I}$ and II methylation status in all cell lines. Briefly, genomic DNA was extracted from the cell lines using the QIAamp DNA Mini kit (Qiagen) and treated with bisulfite to convert all the unmethylated cytosines to uracils while conserving all the methylated cytosines. After treatment, 1- $\mu 1$ aliquots were amplified by PCR. The pyrosequencing primers were as follows: CpGI-F, 5'-GTAAGGGAGAAAGAAGTTAGA-3' and CpGI-R (50-Biotin), 5'-biotin-TA CTATCCTAACAAAA CCCTC-3'; CpGII-F, 5'-GTTGGGTTAGTTTTTTATAGTT GGTT-3' and CpGII-R (50-Biotin), 5'-biotin-AACCAAAC AACCTAAAAAACAAATAC-3'.

The biotinylated $\mathrm{PCR}$ products were purified and subjected to pyrosequencing using the PSQHS 96A Pyrosequencing System and Pyro Gold reagents (PSQ 96MA; Biotage, Charlottesville, VA, USA). A methylation density cut-off point of $15 \%$ was considered significant.

Western blotting. Expression of Ac-STAT3 in the HOSEpiC and HO-8910 cell lines was analyzed by western blotting using commercially available antibodies from Santa Cruz Biotechnology. The cell lysates were subjected to sodium dodecyl sulfate (SDS)-PAGE and subsequently transferred to a PVDF membrane. Blots were visualized using Amersham western blot detection reagent (GE Healthcare). 

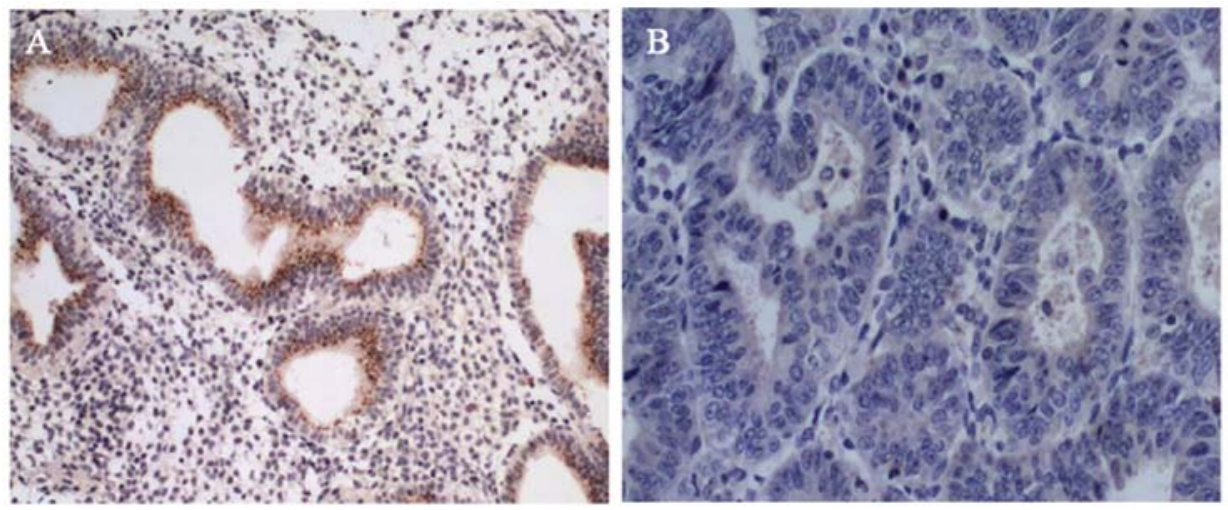

Figure 1. ARHI is downregulated in epithelial ovarian cancer tissue as determined by immunohistochemical staining. (A) ARHI expression in epithelial cells of normal ovarian tissue. (B) ARHI expression in epithelial cells in ovarian cancer tissue.

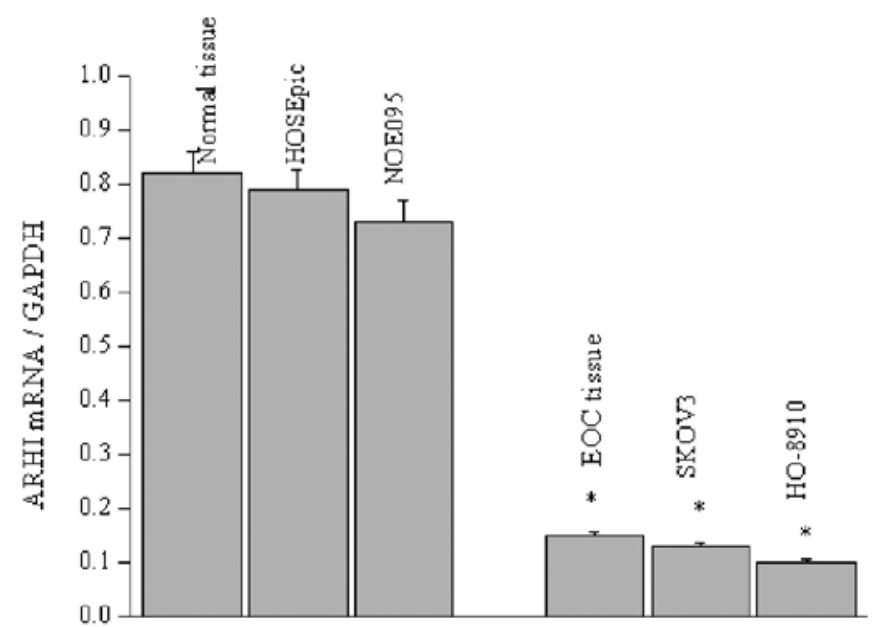

Figure 2. ARHI mRNA is present at a low level in epithelial ovarian cance (EOC) tissues and cells. Results are shown as the means \pm SD. ${ }^{*} \mathrm{P}<0.05$, significant difference is indicated.

Cell proliferation assay. The cells were seeded in 96-well plates $\left(5 \times 10^{4} /\right.$ well) and incubated with 5 -Aza $(0.5-1 \mu \mathrm{M})$ for three days. The culture medium was changed daily to maintain the desired concentration of drugs. The cell proliferation assay was performed using the CyQuant ${ }^{\circledR}$ NF Cell Assay kit (Invitrogen, Carlsbad, CA, USA) following the manufacturer's instructions. Cell viability was measured at a wavelength of $490 \mathrm{~nm}$.

Chromatin immunoprecipitation. ChIP assays were performed as previously described (17) with minor modifications. Briefly, chromatin samples isolated from HO-8910 cells and the STAT3 mutant were sonicated to shear the DNA to an average length of 200-500 bp. Samples were centrifuged and resuspended in dilution buffer, then incubated overnight, at $4^{\circ} \mathrm{C}$ with primary antibody against STAT3 or DNMT1 (3 mg/ml). Protein agarose beads were added to isolate the immune complexes. After being washed from the agarose beads, the DNA-protein crosslinks in the immunopreciptated chromatin complexes were reversed by heating at $65^{\circ} \mathrm{C}$ overnight, and the DNA was eluted. The ARHI DNA in the immunoprecipitates was then detected by PCR using gene-specific primers. Similarly, input was prepared by treating aliquots of chromatin with proteinase
$\mathrm{K}$, heated at $65^{\circ} \mathrm{C}$ for $6 \mathrm{~h}$ for decrosslinking and the resulting DNA was subjected to RT-PCR for amplification. The primers for ARHI amplification were described above. The PCR products were analyzed by electrophoresis on $2 \%$ agarose gels. The signals were normalized as the amount of ARHI DNA in the immunoprecipitate/amount of DNA input.

Immunoprecipitation. Immunoprecipitation assays were performed as previously described (18). Control HO-8910 cells and the mutant STAT3 cells $\left(1 \times 10^{6}\right)$ were incubated for $24 \mathrm{~h}$ and then treated with lysis buffer. For immunoprecipitation, $3 \mathrm{mg}$ of anti-Stat 3 or anti-NMT1 antibody was added to $2 \mathrm{mg}$ of protein lysate. After overnight incubation at $4^{\circ} \mathrm{C}$, protein- $\mathrm{G}$ beads were added and incubated for $1.5 \mathrm{~h}$ at $4^{\circ} \mathrm{C}$. After washing with wash buffer, the immunoprecipitated proteins were eluted for western blotting.

Statistical analysis. Data are expressed as means \pm SD. All statistical analyses were carried out using SPSS 13.0 (SPSS Inc., Chicago, IL, USA). Statistical comparisons were performed by one-way analysis of variance followed by the Student-Newman-Keuls test. $\mathrm{P}<0.05$ was considered to indicate a statistically significant result.

\section{Results}

ARHI expression is downregulated in ovarian cancer cells. ARHI has been identified as a tumor-suppressor gene and is of significant importance in modulating cell growth and apoptosis (19). Consistent with previously conducted epidemiological studies, we found that ARHI expression was strikingly decreased in the majority of ovarian cancer tissues with loss of cell cycle control. Obvious staining for ARHI was present in epithelial cells in normal ovarian tissue sections (Fig. 1A) while almost no detectable expression in cancerous tissues was noted by immunohistochemical staining (Fig. 1B), which was confirmed by RT-PCR measurement. Likewise, the ARHI mRNA level was determined in two normal and ovarian cancer epithelial cell lines, since EOC is clearly responsibility for $90 \%$ of ovarian neoplasias. Similar to the expression observed in the tissue sections, ARHI mRNA levels present were significantly decreased in the SKOV3 and HO-8910 cells, with less mRNA detected in the HO-8910 cells (Fig. 2). 

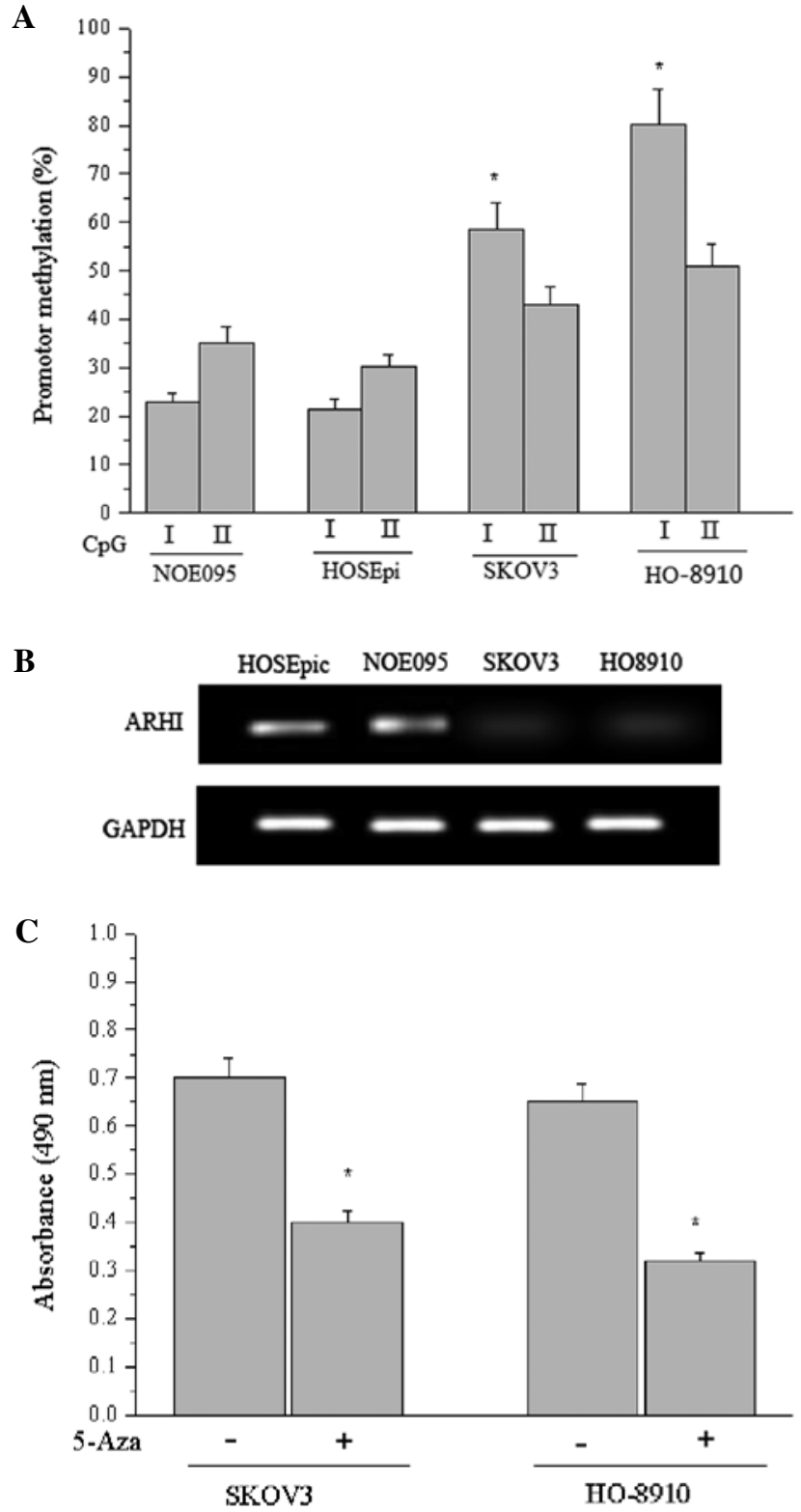

Figure 3. Promoter methylation is involved in ARHI expression and cell growth. (A) ARHI promoter methylation in ovarian cancer cell lines (SKOV3 and HO-8910) and normal ovarian epithelial cell cultures (NOE095 and HOSEpiC) was detected by pyrosequencing analysis. (B) ARHI expression was assessed by RT-PCR. (C) 5-Aza-2'-deoxycytidine (5-Aza) inhibited the proliferation of ovarian cancer cells via demethylation of the ARHI promoter. Data are shown as the means $\pm \mathrm{SD} ;{ }^{*} \mathrm{P}<0.05$.

Promoter methylation is associated with ARHI expression and tumor growth. It has been reported that promoter methylation plays vital roles in the inactivation of the ARHI gene; thus, we examined the methylation status of ARHI. As reported by Luo et al (8), two CpG islands I and II were located within the ARHI promoter and adjacent exon 1. As shown in Fig. 3A, the proportions of methylated $\mathrm{CpG}$ island I in NOE095 and HOSEpiC cells were $22.8 \pm 4.2 \%$ and $21.5 \pm 3.1 \%$, respectively, whereas they were $58.7 \pm 5.4 \%$ and $80.6 \pm 10.2 \%$ in the SKOV3 and HO-8910 cells $(\mathrm{P}<0.05)$, suggesting that $\mathrm{CpG}$ island I in the cancer cells was partially methylated or hypermethylated. Similarly, the density of $\mathrm{CpG}$ island II methylation was increased in EOC cells compared with normal cells. Additionally, the predominate change in

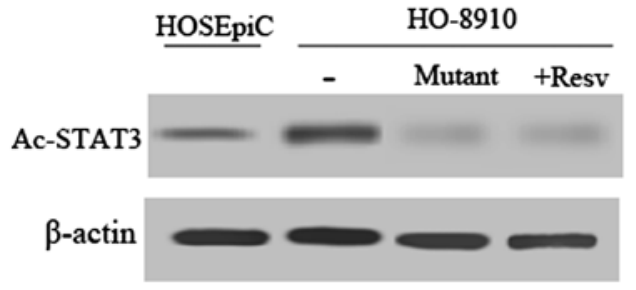

Figure 4. Western blot analysis of acetylated STAT3 in normal ovarian cells (HOSEpiC) and cancer cells (HO-8910). Ac-STAT3 expression was modified by histone deacetylase activator resveratrol (Resv) while STAT3 K685R mutation resulted in downregulation of Ac-STAT3.

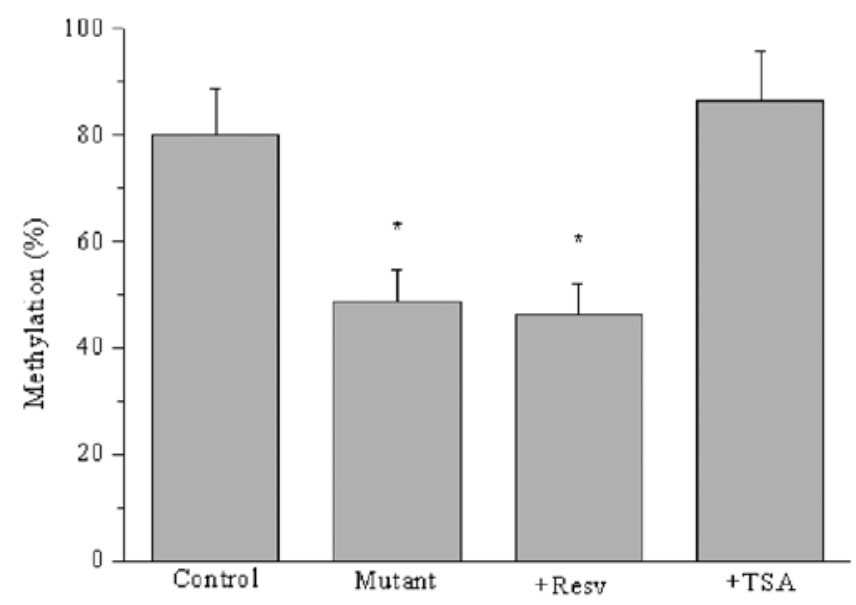

Figure 5. Methylation of ARHI CpG I is regulated by acetylated STAT3. Administration of resveratrol (Resv) or histone deacetylase inhibitor trichostatin (TSA) altered the methylation of the promoter. Data are shown as the means $\pm \mathrm{SD} ;{ }^{*} \mathrm{P}<0.05$.

CpG island I methylation density may shed light on its critical function in regulating ARHI expression. Therefore, RT-RCR was performed to determine the ARHI transcriptional level in each cell line in response to different methylation states, suggesting that the hypermethylated promoter inhibited transcription of ARHI (Fig. 3B). Subsequently, we measured the effect of 5-Aza (5-aza-2'-deoxycytidine) on the viability of SKOV3 and HO-8910 cells using a cell proliferation assay and demonstrated that promoter methylation-mediated inactivation of the tumor-suppressor gene ARHI accounted for the cellular malignances, while 5-Aza-induced demethylation greatly diminished the proliferation of the cancer cells (Fig. 3C).

Elevated STAT3 acetylation results in methylation of the ARHI gene promoter. It has been proposed that STAT3 exerts an influence on $\mathrm{CpG}$ methylation, whereas the underlying mechanisms remain elusive. The present study was carried out to investigate the possible role of acetylated STAT3 in the methylation of $\mathrm{CpG}$ islands. The ARHI gene promoter in the HO-8910 cell line exhibited a higher methylation density than that in the SKOV3 cells; therefore, it was selected for the following studies. Correspondingly, the HO-8910 cell line harboring an endogenous STAT3 mutation with K685 converted to R685 was generated via a homologous recombination-mediated site-directed mutagenesis strategy as previously described (15). First, we assessed the acetylation status of STAT3 by western blot analysis using an Ac-STAT3 
A

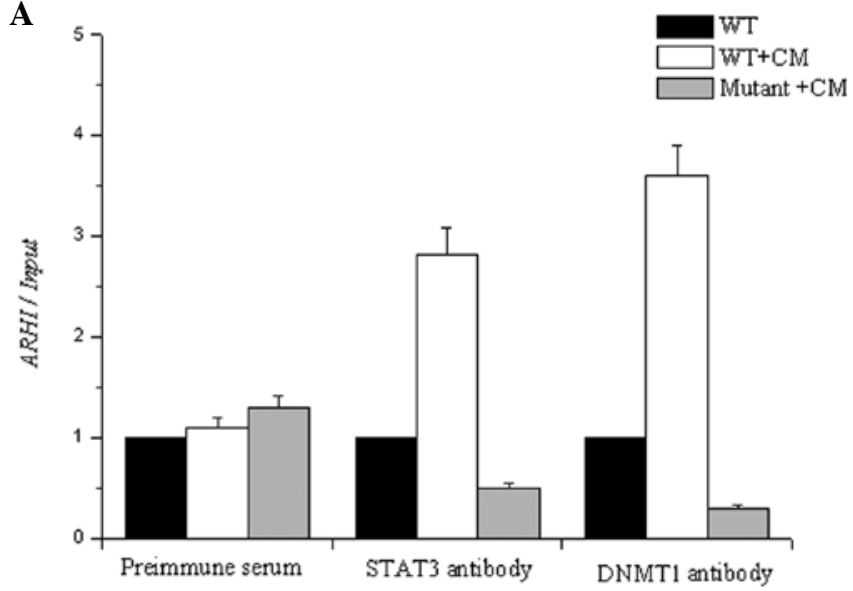

B

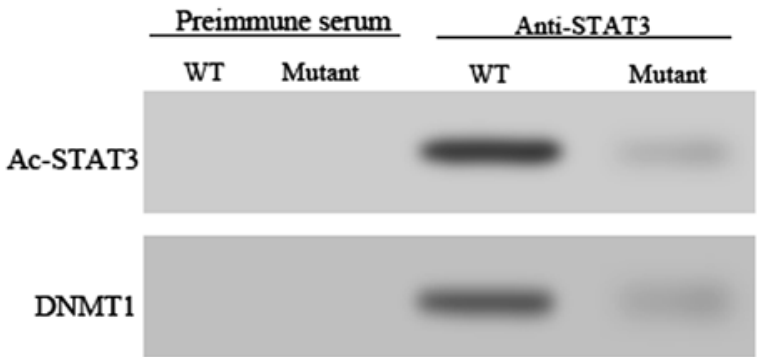

Figure 6. Interaction between acetylated STAT3 and DNMT1 promotes ARHI methylation. (A) Chromatin immunoprecipitation analysis was carried out to detect the recruitment of STAT3 and DNMT1 to the ARHI promoter ARHI presentation in HO-8910 cells incubated with preimmune serum was set as 1. (B) Western blot analysis after immunoprecipitation with the STAT3 antibody to detect the interaction between Ac-STAT3 and DNMT1 in HO-8910 cells (WT) and its corresponding STAT3 K685R mutant cells (mutant).

monoclonal antibody. In comparison with the normal cell line HOSEpiC, STAT3 was relatively highly acetylated in the HO-8910 cells (Fig. 4). Meanwhile, Ac-STAT3 expression decreased sharply after treatment with resveratrol, a histone deacetylase (HDAC) activator or mutation of STAT3 K685R in HO-8910 cells.

Furthermore, we determined whether CpG I island methylation was associated with acetylated STAT3 in HO-8910 cells. As shown in Fig. 5, ARHI promoter methylation was reduced in response to either resveratrol or K685 mutation-mediated decreased Ac-STAT3 $(\mathrm{P}<0.05)$. However, CpG I island methylation was increased by treatment with trichostatin, a histone deacetylase inhibitor that restrains the deacetylation of STAT3. Thus, STAT3 acetylation accelerated the methylation of the ARHI promoter, which regulated the expression of the tumor-suppressor gene ARHI.

STAT3 acetylation mediates STAT3-DNMT1 interaction and recruitment to the gene promoter. It has been reported that binding of inducible transcription factors to DNA helps to recruit chromatin-modification machinery; therefore, chromatin immunoprecipitation was performed to determine whether DNMT1 was attached to ARHI promoter regions in the HO-8910 cell line and the corresponding variant STAT3 K685R cell line. Additionally, tumor-conditioned medium prepared from U251 human glioma cells was applied for the cultivation of HO-8910 and the variant cells to activate STAT3 and facilitate binding to the gene promoter. Furthermore, the binding of DNMT1 to ARHI was also subjected to ChIP assay. As illustrated in Fig. 6A, both STAT3 and DNM1 bound to the ARHI promoter in HO-8910 cells while this binding was reduced in the STAT3 K685 mutant, suggesting that acetylation of STAT3 regulated the attachment of STAT3 to the promoter. Notably, the decreased Ac-STAT3 in the mutant HO-8910 cells prevented the recruitment of DNMT1 to the ARHI gene. We then analyzed the Ac-STAT3-DNMT1 interaction using immunoprecipitation using anti-Ac-STAT3 antibody. Ac-STAT3 was found to interact with DNMT1 (Fig. 6B), which is directly responsible for the modification of the DNA.

\section{Discussion}

ARHI is a maternally imprinted tumor-suppressor gene that is expressed in normal ovarian epithelium and is downregulated in the majority of ovarian cancers. Accumulating evidence has shown that decreased ARHI expression, either eliminated by $\mathrm{LOH}$ or post-transcriptional modulation, is implicated in tumor proliferation and development (20). Our study was carried out to investigate whether methylation of the ARHI promoter gene could mute its expression and the underlying mechanism of ARHI methylation.

The present study also revealed that ARHI was downregulated in EOC as measured by IHC. To the best of our knowledge, ARHI possesses three $\mathrm{CpG}$ islands that are prone to methylation. CpG I and II are located within promoter regions, which encouraged us to consider the potential role of promoter methylation in the regulation of ARHI expression. In comparison with normal ovarian epithelium cells, the ARHI promoter was partially or heavily methylated in cancer cells in concert with reduced ARHI expression. Additionally, we demonstrated that $\mathrm{CpG}$ I hypermethylation suppressed ARHI promoter activity and regulated ARHI expression in ovarian cancer cells, which is similar to the outcomes obtained in breast cancer cells.

DNA methylation acts in a switch-like manner and is strongly correlated with the absence of gene expression and low levels of additional activating epigenetic markers (21). Although the well-established role of DNA methylation in gene silencing has been extensively investigated, the precise mechanism of how the promoter methylation status is modified remains elusive. It has been reported that binding of inducible transcription factors to DNA helps recruit chromatin-modification machinery, which is responsible for the modification and expression of DNA (22). Furthermore, STAT3 has been shown to increase $\mathrm{CpG}$ island methylation when acetylated (23). Therefore, we explored whether STAT3 was associated with the methylation-induced ARHI silencing. It was confirmed that acetylated STAT3 bound to the ARHI promoter regions and recruited DNMT1 to suppress ARHI expression. Simultaneously, we found that specific inhibition of STAT3 acetylation using resveratrol reversed the suppression of ARHI and restrained cancer cell growth.

Taken together, our study demonstrated that the tumorsuppressor ARHI is downregulated in ovarian epithelium cancer cells and this downregulation can be partially ascribed 
to the hypermethylation of CpG I islands. Acetylated STAT3 bound and recruited DNMT1 to ARHI promoter regions. The activation of histone deacetylase or inhibition of acetyltransferase may hold promise for eliminating methylation-induced tumor-suppressor silencing, and further prospective studies are warranted to further clarify the modification machinery of Ac-STAT.

\section{Acknowledgements}

The present research was supported by a grant from the Natural Science Foundation of China (no. 39830350).

\section{References}

1. Yu Y, Xu F, Peng H, et al: NOEY2 (ARHI), an imprinted putative tumor suppressor gene in ovarian and breast carcinomas. Proc Natl Acad Sci USA 96: 214-219, 1999.

2. Peng H, Xu F, Pershad R, et al: ARHI is the center of allelic deletion on chromosome $1 \mathrm{p} 31$ in ovarian and breast cancers. Int $\mathrm{J}$ Cancer 86: 690-694, 2000.

3. Luo RZ, Fang X, Marquez R, et al: ARHI is a Ras-related small G-protein with a novel N-terminal extension that inhibits growth of ovarian and breast cancers. Oncogene 22: 2897-2909, 2003.

4. Zhao X, Li J, Zhuo J and Cai L: Reexpression of ARHI inhibits tumor growth and angiogenesis and impairs the mTOR/VEGF pathway in hepatocellular carcinoma. Biochem Biophys Res Commun 403: 417-421, 2010.

5. Zou CF, Jia L, Jin H, et al: Re-expression of ARHI (DIRAS3) induces autophagy in breast cancer cells and enhances the inhibitory effect of paclitaxel. BMC Cancer 11: 22, 2011.

6. Yang H, Lu X, Qian J, et al: Imprinted tumor suppressor gene ARHI induces apoptosis correlated with changes in DNA methylation in pancreatic cancer cells. Mol Med Rep 3: 581-587, 2010 .

7. Kobayashi H, Kajiwara H, Kanayama S, et al: Molecular pathogenesis of endometriosis-associated clear cell carcinoma of the ovary (Review). Oncol Rep 22: 233-240, 2009.

8. Luo RZ, Peng H, Xu F, et al: Genomic structure and promoter characterization of an imprinted tumor suppressor gene ARHI. Biochim Biophys Acta 1519: 216-222, 2001.

9. Feng W, Marquez RT, Lu Z, et al: Imprinted tumor suppressor genes ARHI and PEG3 are the most frequently down-regulated in human ovarian cancers by loss of heterozygosity and promoter methylation. Cancer 112: 1489-1502, 2008.

10. Yuan Z, Guan Y, Chatterjee D and Chin YE: Stat3 dimerization regulated by reversible acetylation of a single lysine residue. Science 307: 269-273, 2005.
11. Wang R, Cherukuri P and Luo J: Activation of Stat3 sequencespecific DNA binding and transcription by $\mathrm{p} 300 / \mathrm{CREB}-$ binding protein-mediated acetylation. J Biol Chem 280: 11528-11534, 2005.

12. Kimura K, Yamada T, Matsumoto M, et al: Endoplasmic reticulum stress inhibits STAT3-dependent suppression of hepatic gluconeogenesis via dephosphorylation and deacetylation. Diabetes 61: 61-73, 2012.

13. Morgan RJ, Alvarez RD, Armstrong DK, et al: Epithelial ovarian cancer. J Natl Compr Cancer Netw 9: 82-113, 2011.

14. Higashiura Y, Kajihara H, Shigetomi $\mathrm{H}$ and Kobayashi H: Identification of multiple pathways involved in the malignant transformation of endometriosis (Review). Oncol Lett 4: 3-9, 2012.

15. Lee H, Zhang P, Herrmann A, et al: Acetylated STAT3 is crucial for methylation of tumor-suppressor gene promoters and inhibition by resveratrol results in demethylation. Proc Natl Acad Sci USA 109: 7765-7769, 2012

16. Yuan J, Luo RZ, Fujii S, et al: Aberrant methylation and silencing of ARHI, an imprinted tumor suppressor gene in which the function is lost in breast cancers. Cancer Res 63: 4174-4180, 2003.

17. Sharda DR, Yu S, Ray M, et al: Regulation of macrophage arginase expression and tumor growth by the Ron receptor tyrosine kinase. J Immunol 187: 2181-2192, 2011

18. Badgwell DB, Lu Z, Le K, et al: The tumor-suppressor gene ARHI (DIRAS3) suppresses ovarian cancer cell migration through inhibition of the Stat 3 and FAK/Rho signaling pathways. Oncogene 31: 68-79, 2012.

19. Bao JJ, Le XF, Wang RY, et al: Reexpression of the tumor suppressor gene ARHI induces apoptosis in ovarian and breast cancer cells through a caspase-independent calpain-dependent pathway. Cancer Res 62: 7264-7272, 2002.

20. Lu Z, Luo RZ, Peng H, et al: Transcriptional and post-transcriptional down-regulation of the imprinted tumor suppressor gene ARHI (DRAS3) in ovarian cancer. Clin Cancer Res 12: 2404-2413, 2006.

21. Qidwai MT, Jamal F, Singh D and Sharma RK: Factors modifying transcriptional regulation of signaling genes have putative role in tumor development and progression in humans. Med Hypotheses 79: 805-812, 2012

22. Stark GR, Wang Y and Lu T: Lysine methylation of promoterbound transcription factors and relevance to cancer. Cell Res 21: 375-380, 2011.

23. Zhang Q, Wang HY, Marzec M, Raghunath PN, Nagasawa T and Wasik MA: STAT3- and DNA methyltransferase 1-mediated epigenetic silencing of SHP-1 tyrosine phosphatase tumor suppressor gene in malignant T lymphocytes. Proc Natl Acad Sci USA 102: 6948-6953, 2005. 